\title{
Improvement in automated detection of pulmonary nodules on helical x-ray CT images
}

\author{
Yongbum Lee $^{* a}$, Du-Yih Tsai ${ }^{\mathrm{a}}$, Takeshi Hara ${ }^{\mathrm{b}}$, Hiroshi Fujita ${ }^{\mathrm{b}}$, Shigeki Itoh ${ }^{\mathrm{c}}$, Takeo Ishigaki ${ }^{\mathrm{d}}$ \\ ${ }^{a}$ School of Health Sciences, Niigata University, 2-746 Asahimachi-dori, Niigata, 951-8518 Japan \\ ${ }^{\mathrm{b}}$ Graduate School of Medicine, Gifu University, 1-1 Yanagido, Gifu, 501-1193 Japan \\ ${ }^{c}$ School of Health Sciences, Nagoya Univ., 1-1-20 Taikominami, Higashi-ku, Nagoya, 461-8673 Japan \\ ${ }^{\mathrm{d}}$ Nagoya University School of Medicine, 65 Turumai-cho, Shouwa-ku, Nagoya, 466-8550 Japan
}

\begin{abstract}
We previously developed a scheme to automatically detect pulmonary nodules on CT images, as a part of computeraided diagnosis (CAD) system. The proposed method consisted of two template-matching approaches based on simple models that simulate real nodules. One was a new template-matching technique based on a genetic algorithm (GA) template matching (GATM) for detecting nodules within the lung area. The other one was a conventional template matching along the lung wall [lung wall template matching (LWTM)] for detecting nodules on the lung wall. After the two template matchings, thirteen feature values were calculated and used for eliminating false positives. Twenty clinical cases involving a total of 557 sectional images were applied; 71 nodules out of 98 were correctly detected with the number of false positives at approximately 30.8/case by applying two template matchings (GATM and LWTM) and elimination process of false positives. In this study, five features were newly added, and threshold-values of our previous features were reconsidered for further eliminating false positives. As the result, the number of false positives was decreased to $5.5 /$ case without elimination of true positives.
\end{abstract}

Keywords: computer-aided diagnosis (CAD), computed tomography (CT), pulmonary nodule detection, template matching, genetic algorithm (GA), false positive elimination

\section{INTRODUCTION}

Computed tomography (CT) can help detect lung cancer at early stages because through this, it is possible to visualize small or low-contrast lung nodules that could barely be seen on conventional radiograms. However, a single CT examination has to interpret approximately 30 section images for $10-\mathrm{mm}$ reconstruction intervals. Evaluating all of them must prove exhausting for radiologists. Therefore, some schemes have been developed to automatically detect pulmonary nodules on CT images, as a part of computer-aided diagnosis (CAD) system [1-12]. Of those, we have developed a nodule detection method based on improved template-matching techniques using simple models that simulated real nodules [4-7]. Candidates detected by the template matchings were classified by thirteen features as true positives (TPs) and false positives (FPs). By the scheme, although it was possible to detect typical spherical, circular and semicircular nodules correctly, it was difficult to detect some nodules such as with low contrast, located in the apex and basis pulmonis with artifact, and so on. Also, the remaining number of FPs was over 30 per case when the sensitivity was approximately $72 \%$. To improve the performance, new processes to detect nodules in the difficult cases and to further decrease FPs are required. Therefore, in this study, we mainly focus on false positive elimination. In order to further eliminate FPs, we add five features and reconsider threshold-values of features been used in our previous work. In this paper, first, outline of our proposed detection scheme is described. After that, the details of improved process for further elimination of FPs are presented as well.

\section{OUTLINE OF DETECTION SCHEME}

We previously developed a new template-matching technique based on a genetic algorithm template matching (GATM) for detecting nodules within the lung area $[4,6]$, and developed a conventional template matching along the lung wall [lung wall template matching (LWTM)] to detect nodules that attached to the lung wall [5]. For the template 


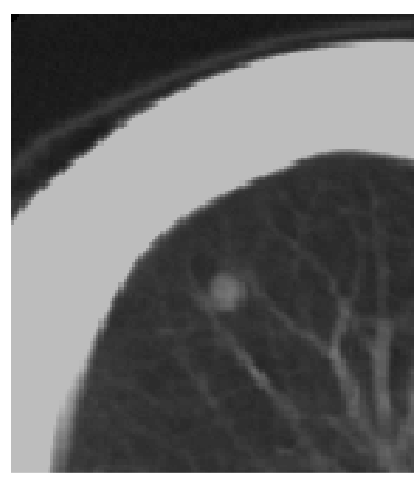

(a).

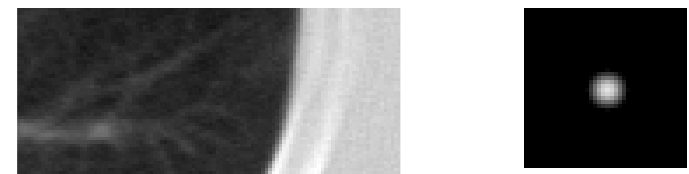

Diameter 10

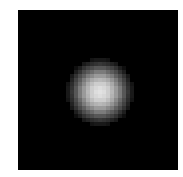

20

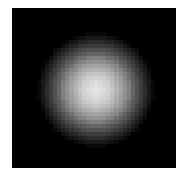

30

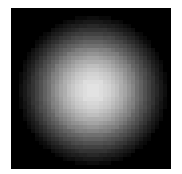

40 pixels

Fig.2 Reference images of circular models for GATM.

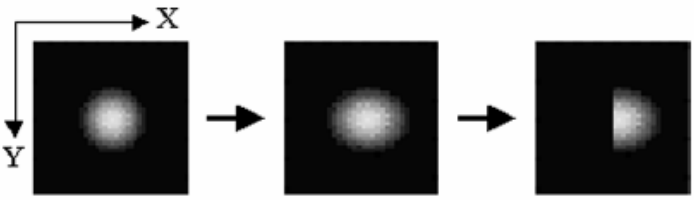

(a) (b) (c)
Fig.3 Making process of reference image used in LWTM. (a) Circular model which diameter is 20 pixels. (b) Model formed by increasing the x-directional length of (a) by $20 \%$. (c) Semicircular model formed by dividing (b).

matchings, simple nodular models that simulate real nodule were used as reference images. After the template matchings, thirteen features were used to classify false positives.

Here, nodules within the lung area tend to have spherical shapes [Fig.1(a)]. In genera, smaller nodules only appear in one slice of a CT scan. However, larger nodules can be found in a set of continuous slices. Nodules on the lung wall also appear in one or more slices, depending on their size. However, they are semicircular in shape [Fig.1(b)]. We observed that the nodules' CT values followed Gaussian-like distribution by investigating the CT values in and around the nodules. Therefore, we used nodular models with Gaussian-like distribution as reference images for our two template-matching methods. The nodular models were determined by the following formula:

$$
p v_{x, y, z}=m \cdot e^{-\left(x^{2}+y^{2}+k \cdot z^{2}\right) / n}
$$

where $p v_{x, y, z}$ is the pixel value of coordinate $(x, y, z)$, and $m$ and $n$ are parameters representing the maximum value and variance of the distribution, respectively. The value $k$ regulates the scaling in $z$. Four spherical models and four circular models (Fig.2) were generated for GATM. The diameters of the models were 10, 20, 30 and 40 pixels (one pixel $=0.68$ $\mathrm{mm}$ ), respectively. Spherical models consist of three continuous slices. The GATM was performed twice, once using the spherical models and once using the circular models as reference images. Two semicircular nodular models were also generated from two circular models, as shown in Fig.3(c), for LWTM. The diameters of these models were 10 and 20 pixels.

Fig.4 illustrates the GATM method using spherical nodular models [4,6]. In the GATM, the GA was used to determine the target position in an observed image and to select an adequate template image from reference images for template matching. So, each individual in our GA has a chromosome with data for determining a location with the 3-D space of chest helical CT images (observed image) and for selecting an adequate reference image. A chromosome is represented in binary digits and consists of a gene. An example of a chromosome is shown in Fig.5. Each chromosome has 25 bits, of which 23 determine the target position and two select the reference image. Furthermore, the 23 position bits are divided into 9-, 9-, and 5-bit sets corresponding to the coordinates $(x, y, z)$. The chromosome shown in Fig.5 selected the reference image having the value of $s=2$ and achieved fitness through a template matching on the coordinate $(x, y, z)=(113,389,14)$ in the observed image. We defined the fitness of the individual as the similarity calculated by the cross-correlation coefficient, as

$$
\text { Similarity }_{a, b}=\frac{\sum_{i=0}^{n-1}\left(a_{i}-m_{a}\right)\left(b_{i}-m_{b}\right)}{\sqrt{\sum_{i=0}^{n-1}\left(a_{i}-m_{a}\right)^{2}} \sqrt{\sum_{i=0}^{n-1}\left(b_{i}-m_{b}\right)^{2}}}
$$




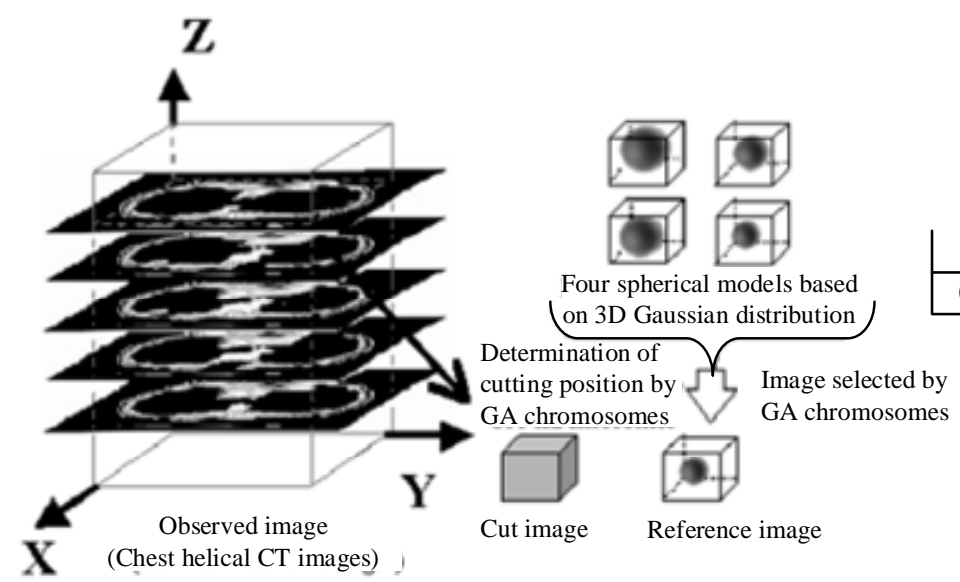

Fig.4 Illustration of GATM using spherical nodular model $[4,6]$.

\begin{tabular}{|c|c|c|c|}
$x$ & $y$ & $z$ & $s$ \\
\hline 001110001 & 110000101 & 01110 & 10 \\
\hline
\end{tabular}

$(x, y, z, s)=(113,389,14,2)$

$\mathrm{x}$ : 9 bits (Max value 512)

$y: 9$ bits (Max value 512)

$z$ : 5 bits (Max value 32)

$x$ : 2 bits (Max value 4)

Fig.5 An example of a chromosome.

where

$$
m_{a}=\frac{1}{n} \sum_{i=0}^{n-1} a_{i}, \quad m_{b}=\frac{1}{n} \sum_{i=0}^{n-1} b_{i}
$$

The cross-correlation coefficient varies from -1 to 1 . The values $a$ and $b$ signify the images for comparison. The value $n$ is the number of pixels in the images. The value $a_{i}$ is the $i$ th pixel in image $a$, the value $b_{i}$ is the $i$ th pixel in image $b$. The cross-correlation coefficient does not depend on the CT values but only the shape of the pixel value distribution in images $a$ and $b$ according to the denominator. In the GA process, first, the initial population was randomly generated from a sequence of zeroes and ones. Next, the fitness of each individual was calculated. Then, sharing was applied. Sharing is a method that makes it possible to obtain multiple solutions by decreasing the fitness of a point after a sufficient number of individuals have gathered around it. It is an effective method for executing the GA in parallel as well as for detecting objects with different sizes or shapes. All individuals in the GA were subjected to genetic operations (i.e., selection, crossover and mutation). The individual population was sorted by fitness value. The half that had the lower fitness values were selected and replaced by new individuals had been crossovered with the half having the higher values. Mutation was executed as a bit inversion. The probability of mutation was $10 \%$ for each bit in the chromosome. The ten individuals having the highest fitness values in a generation avoided the mutation. The individual with the highest fitness in each generation was continuously carried over to the next generation regardless of any conditions. The process of fitness calculation, sharing and genetic operations constitute one generation. The initial population consisted of 124 individuals, and the maximum number of generations was 200 .

LWTM process is shown in Fig.6. In the LWTM, first, the rough lung area was extracted using pixel thresholding and labeling techniques. A rectangle encompassing the extracted lung areas was determined. We defined the contour of the lung wall as the first dot that the scanner encountered on the enclosed area as it scanned from each edge of the rectangle toward the lung area. An example of extracted contour of the lung wall is shown in Fig. 7. Then, template matching was executed along the contour of the lung wall using the semicircular model that rotated corresponding to the angle tangent to the lung wall. The similarity was calculated by the correlation coefficient indicated in (2).

After the template matchings, nine features in the GATM and four features in the LWTM were used to classify false positive candidates. The features and their tendencies are shown in Fig 8. These features were calculated in regions of interest (ROIs) including the candidate. A mean and standard deviation $(S d)$ were calculated from all pixels in ROIs (40x40 pixels). In our investigating, we found that these FPs had higher overall CT values that varied greater than those of the nodules candidates. Area, circularity (Cir) and irregurarity (Irr) were calculated in candidate region. Irregularity was defined as the standard deviation of the distance form the center of the candidate region to the edge. The candidate region was extracted automatically by calculating these features while changing the threshold value (Thv) of the pixel thresholding and labeling techniques. The initial Thv was set to the mean CT value (Mctv) of the $3 \times 3$ pixel grid that 


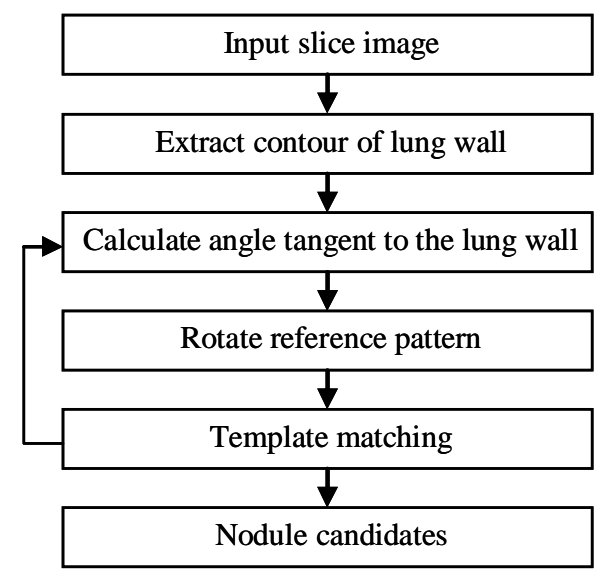

Fig.6 Procedural flowchart of LWTM

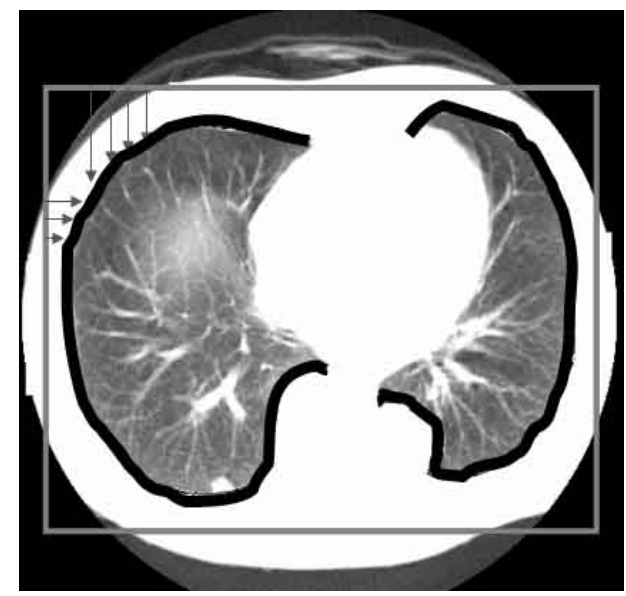

Fig.7 Rectangle encompassing the lung area and extracted contour of the lung wall.

\begin{tabular}{|c|c|c|}
\hline Target & Feature & Tendency \\
\hline $\begin{array}{l}\text { Candidates } \\
\text { detected by } \\
\text { GATM }\end{array}$ & $\begin{array}{l}\text { Mean } \\
\text { Standard deviation }(S d) \\
\text { Area } \\
\text { Circularity (Cir) } \\
\text { Irregularity (Irr) } \\
\text { Contrast (Cont) } \\
\text { Max mean CT value }(M m c t) \\
\text { Directional variance } \\
\text { of pixel gradient ( } D v p g) \\
\text { Directional cross-correlation } \\
\text { of pixel gradient }(D c p g)\end{array}$ & $\begin{array}{l}\mathrm{TP}<\mathrm{FP} \\
\mathrm{TP}<\mathrm{FP} \\
\mathrm{P}<\mathrm{TP}<\text { large FP } \\
\mathrm{TP}>\mathrm{FP} \\
\mathrm{TP}<\mathrm{FP} \\
\mathrm{TP}<\mathrm{FP} \\
\mathrm{TP}<\mathrm{FP} \\
\mathrm{TP}>\mathrm{FP} \\
\mathrm{TP}>\mathrm{FP}\end{array}$ \\
\hline $\begin{array}{l}\text { Candidates } \\
\text { detected by } \\
\text { LWTM }\end{array}$ & $\begin{array}{l}\text { Inverse difference moment }(I d m) \\
\text { Entropy }(\text { Ent }) \\
\text { Area } \\
\text { Contrast }(\text { Cont })\end{array}$ & $\begin{array}{l}\mathrm{TP}<\mathrm{FP} \\
\mathrm{TP}>\mathrm{FP} \\
\mathrm{P}<\mathrm{TP}<\text { large } \mathrm{FP} \\
\mathrm{TP}<\mathrm{FP}\end{array}$ \\
\hline
\end{tabular}

(a)

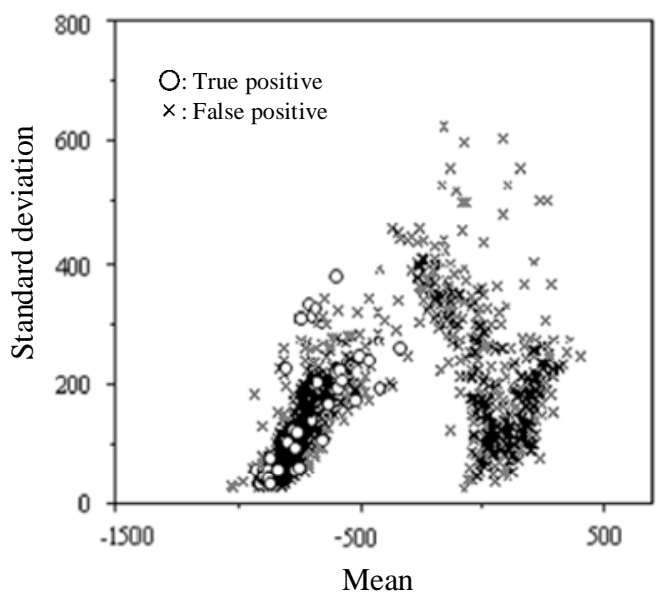

(b)

Fig.8 Thirteen features used to classify FPs and an example of distribution of feature values. (a) Tendencies exhibited by the features of TPs and FPs. (b) Distribution of standard deviation versus mean.

includes the center pixel of the ROI (100x100 pixels) minus 200. The area, circularity and irregularity were calculated while adding 20 to $T h v$, and when those feature values were in a specific range, the region was declared a candidate region and the candidate was deemed a TP. When $T h v>M c t v+60$, the candidate was deemed a FP. An example of TP candidate region is shown in Fig.9. In the Fig.9, the candidate region was determined when Thv $=$ Mctv -120 . The determined candidate regions were also used to calculate contrast (Cont) and max mean CT value (Mmct). Contrast was defined as the difference $(|M c-M n|)$ between the mean CT values in the candidate region $(M c)$ [Fig.10(a)] and the neighboring region $(\mathrm{Mn})$ [Fig.10(b)] enclosing it. If this value is high, the candidate has marked contrast and is deemed a FP. Here, the neighboring region was extracted by expanding the size of the candidate region. In addition, the $M m c t$ was defined as the mean value of the five maximum pixels in the candidate region. If Mmct is also high, the candidate is deemed a FP.

Nodules tend to have pixel gradients that radiate from the center out. Some of the smaller FPs consisted of diverged or intersected blood vessels. The direction of the pixel gradient in the surrounding region of such FP candidates varied 


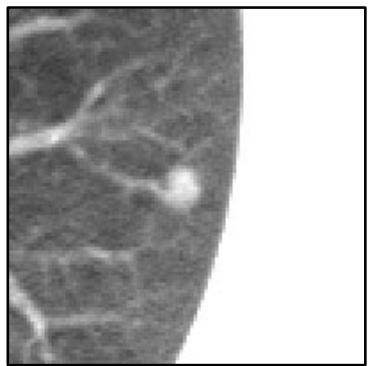

(a)

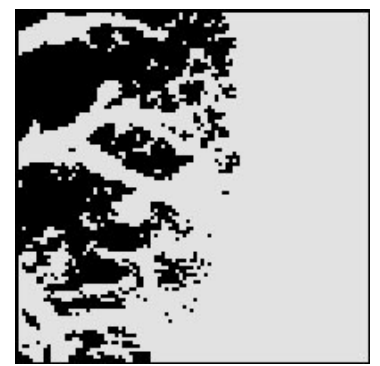

(b)

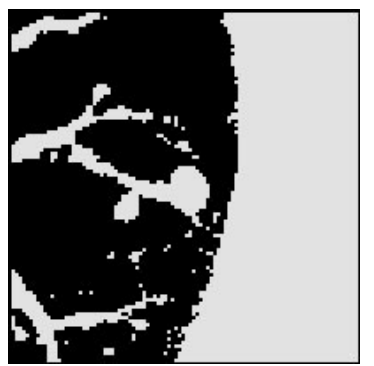

(c)

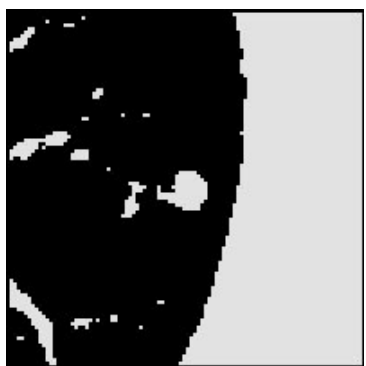

(d)

Fig.9 Example of TP candidate and threshold images at each step. (a) Original image (100x100 pixels). (b) Threshold image when $T h v=M c t v-200$. (c) Threshold image when Thv=Mctv-160. (d) Threshold image when Thv=Mctv-120. Determined candidate region.

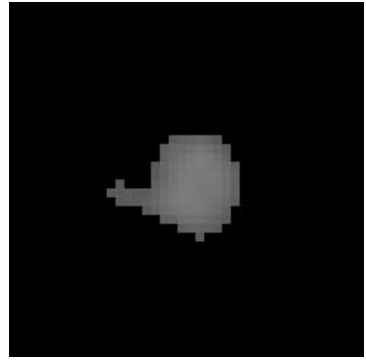

(a)

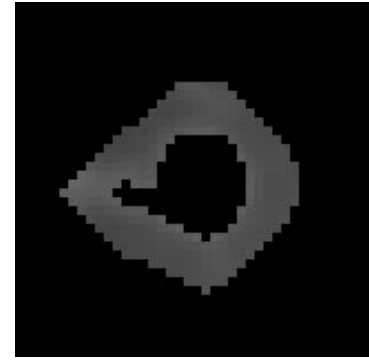

(b)

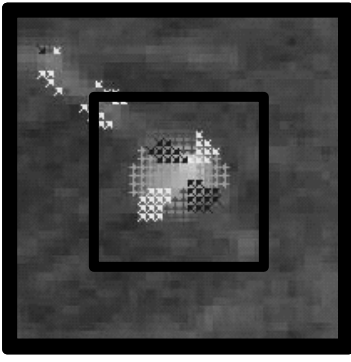

(a)

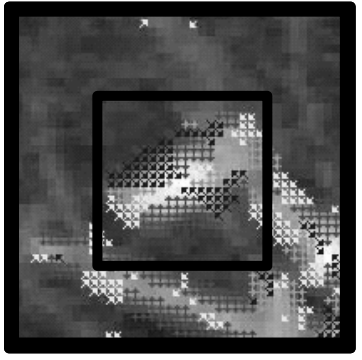

(b)

Fig.10 Candidate region and the neighboring region for contrast calculation. (a) Candidate region. (b) Neighboring region.

Fig.11 Candidate images (40x40 pixels) with the directions of pixel gradients indicated tiny arrows. Dvpg $g_{\text {inn }}$ is calculated in small rectangle region. Dvpg out is calculated in large rectangle region. (a) A TP candidate, $D v p g_{\text {inn }}=246.8$, $D v p g_{\text {out }}=146.0$. (b) A FP candidate, $D v p g_{\text {inn }}=273.2$, Dvpg $_{\text {out }}=240.5$.

more than that of TPs. So by comparing the difference $\left(\left|D v p g_{\text {Inn }}-D v p g_{\text {out }}\right|\right)$ in the directional variance of the pixel gradient in a 20x20 pixel region $\left(D v p g_{\text {Inn }}\right)$ and that in a 40x40 pixel region $\left(D v p g_{\text {out }}\right)$, FPs could be classified. In our scheme, Kirsch's filter was used to obtain the direction and intensity of the pixel gradient. Fig. 11 shows the images executed Kirsch's filter and regions for calculation of the directional variance. It should be noted that the directional variance was calculated only if the gradient intensity was greater than an experimentally determined constant. In addition, by correlating of the pixel gradient in the detected candidate and the nodular reference model, FPs can be eliminated. Circular model that diameter is 40 pixels, shown in Fig.2, was used as nodular reference model. Kirsch's filter was used here once more to provide a direction without limiting the intensity of the gradient. To calculate the correlation of the pixel gradients, the (2) was also employed by replacing the pixel values $a_{i}$ and $b_{i}$ with the pixel gradient values.

We focused on the candidates' texture and used the co-occurrence matrix $[Q(i, j)]$, which is well known as one of the typical methods in texture analysis to eliminate FPs. Several features can be calculated from the co-occurrence matrix, such as the angular second moment, entropy (Ent), inverse difference moment (Idm) and contrast. As we observed in our experiment, the Idm and Ent were more effective than other features in decreasing the number of FPs. Their definition is as follows:

$$
I d m=\sum_{i, j} \frac{Q(i, j)}{1+(i-j)^{2}}
$$




\begin{tabular}{|l|l|}
\hline \multicolumn{1}{|c|}{ Target } & \multicolumn{1}{c|}{ Feature } \\
\hline $\begin{array}{l}\text { Candidates } \\
\text { detected by } \\
\text { GATM }\end{array}$ & $\begin{array}{l}\text { Second mean }(\text { Scm }) \\
\text { Second area (Scar) }\end{array}$ \\
\hline $\begin{array}{l}\text { Candidates } \\
\text { detected by } \\
\text { LWTM }\end{array}$ & $\begin{array}{l}\text { Local mean }(\text { Lm }) \\
\text { Local standard deviation }(\text { Lsd }) \\
\text { Local directional variance } \\
\text { of pixel gradient }(\text { Ldvpg })\end{array}$ \\
\hline
\end{tabular}

Fig.12 Added new features for FP elimination.

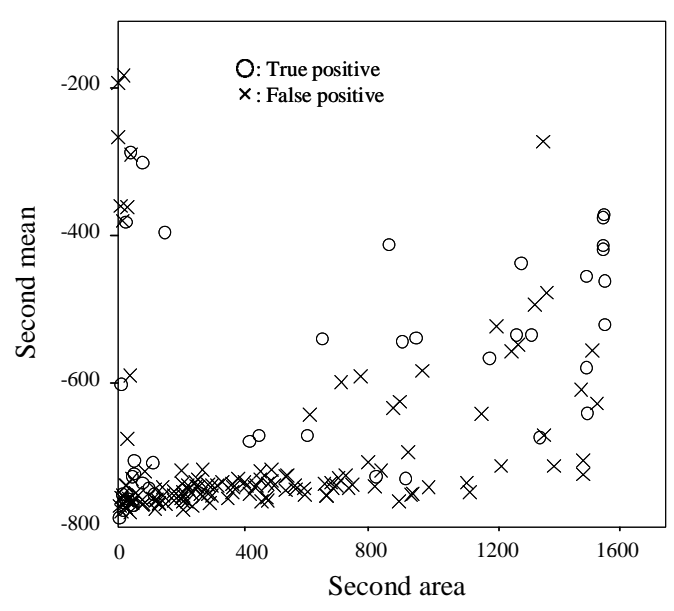

(a)
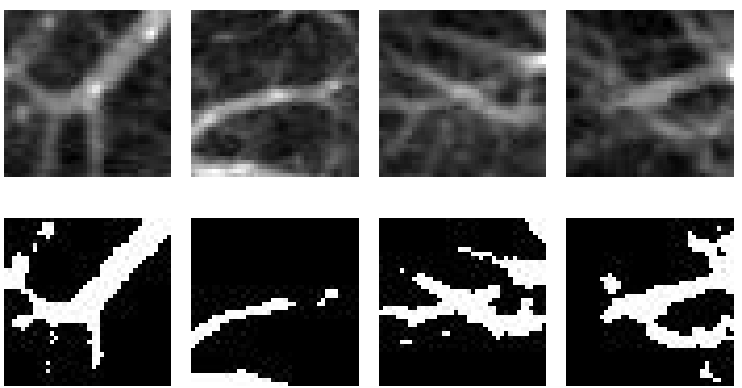

Fig.13 Eliminated FPs by second mean and second area. Upper indicate original images of FPs. Lower indicate threshold images to extract candidate region.

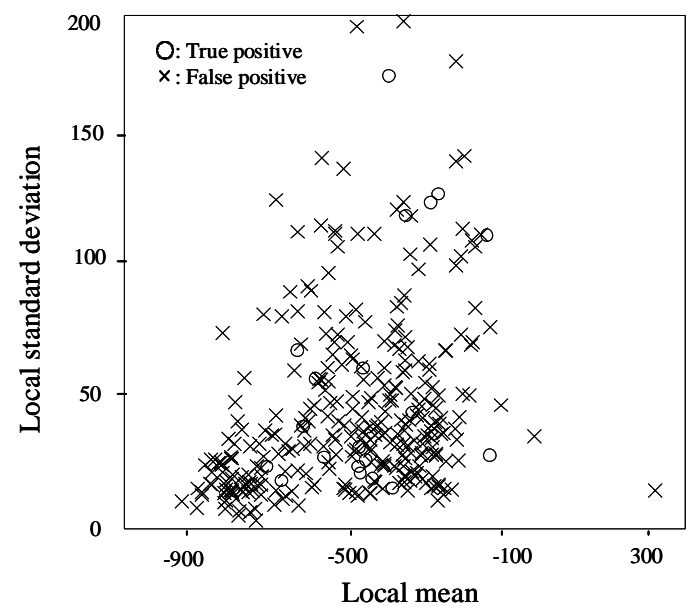

(b)

Fig.14 Distributions of new features. (a) Distribution of second mean verses second area. (b) Distribution of local standard deviation and local mean.

$E n t=-\sum_{i, j} Q(i, j) \cdot \log Q(i, j)$

In (3), the $I d m$ is large if the difference between $i$ and $j$ is small in the matrix, such as in an image with flat pixel distribution. In (4), the Ent is large if the constituent values of the matrix tend to be equal. The Ent signifies the degree of variance in the image's pixel values. By using these texture features, it is possible to eliminate FPs that are difficult extract form the candidate region on the lung wall. In addition, the area and contrast of the candidate region were used to eliminate FPs. The candidate region was determined by the pixel thresholding technique after removing the lung wall area.

\section{IMPROVEMENT IN FP ELIMINATION PROCESS}

Our FPs elimination process was improved by adding five features and by reconsidering threshold-values of features described in Section 2. The newly added features are show in Fig. 12.

Second mean $(\mathrm{Scm})$ and second area (Scar) were applied to the candidates detected by the GMTM. They used to eliminate FPs that could not be eliminated by area, circularity and irregularity. Those FPs tend to be a part of vessels as shown in Fig.13. The overall shape of vessels could not be extracted in the previous changeable-thresholding technique as shown in Fig.9. Therefore, in this process, the constant-threshloding value was used to extract candidate regions 


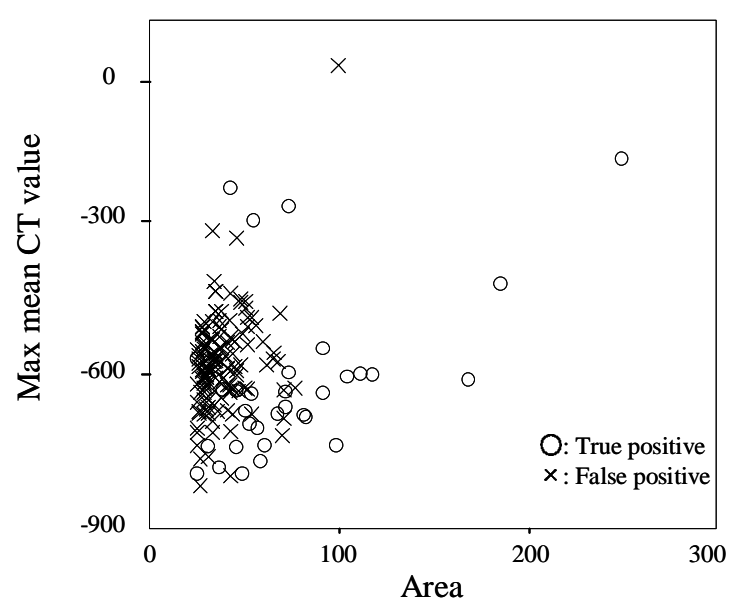

(a)

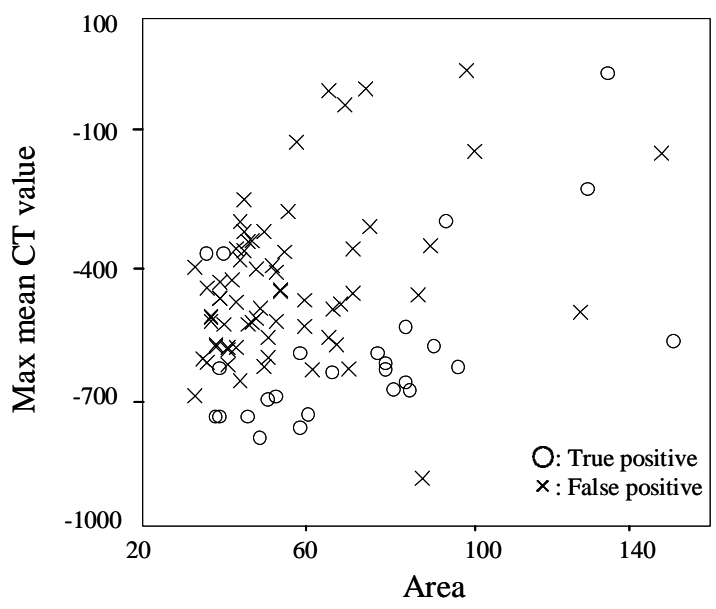

(b)

Fig.15 Distributions of max mean CT value verses area. (a) Distribution of candidates detected by the GATM using circular models. (b) Distribution of candidates detected by the GATM using spherical models.
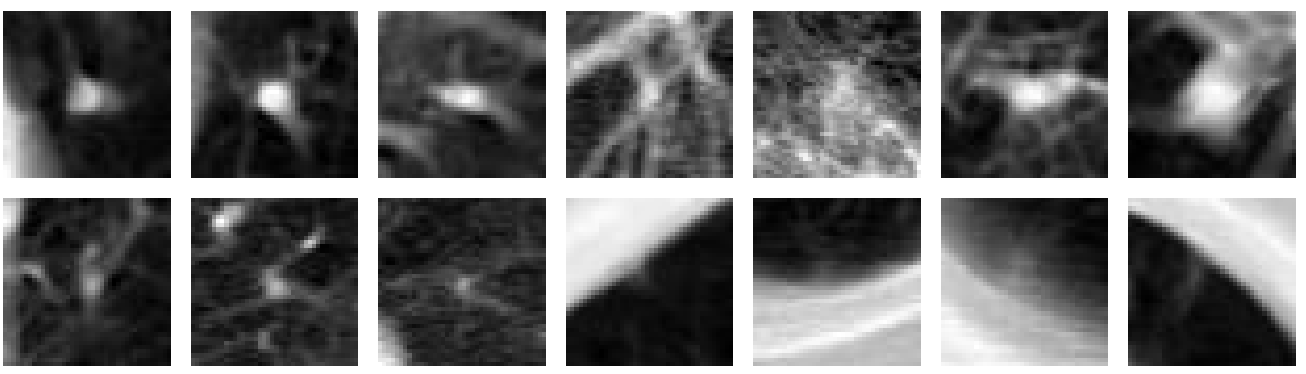

Fig.16 Examples of eliminated FPs in this study.

Table 1 Previous result. Detection rate in term of method and size in mm (successfully detected/total count) and the number of FPs (ratio of FPs/case)

\begin{tabular}{ccccccc}
\hline \hline \multicolumn{3}{c}{ True positives } & & \multicolumn{2}{c}{ False positives } \\
\hline \hline $\begin{array}{c}\text { Diameter }(\mathrm{mm}) \\
\text { /method }\end{array}$ & $<10$ & $10 \sim 20$ & $>20$ & Total & Before FPs elimination & After FPs elimination \\
\hline GATM & $35 / 51$ & $13 / 16$ & $6 / 7$ & $54 / 74$ & $161.2(3223 / 20)$ & $16.7(333 / 20)$ \\
\hline LWTM & $10 / 15$ & $6 / 7$ & $1 / 2$ & $17 / 24$ & $96.5(1930 / 20)$ & $14.2(283 / 20)$ \\
\hline Total & $45 / 66$ & $19 / 23$ & $7 / 9$ & $71 / 98$ & $257.7(5153 / 20)$ & $30.8(616 / 20)$ \\
\hline \hline
\end{tabular}

Table 2 Improved result of \# of FPs (ratio of FPs/case).

\begin{tabular}{lccc}
\hline \hline \multicolumn{1}{c}{ Improvement matters } & \# of FPs by GATM & \# of FPs by LWTM & Total \\
\hline $\begin{array}{l}\text { GATM: Reconsidering of threshold-value } \\
\text { LWTM: Using } L m \text { and } L s d\end{array}$ & $8.0(160 / 20)$ & $3.5(70 / 20)$ & $11.5(230 / 20)$ \\
\hline $\begin{array}{l}\text { GATM: Using } S c m \text { and } S c a r \\
\text { LWTM: Using } L d v p g\end{array}$ & $2.9(58 / 20)$ & $2.6(51 / 20)$ & $5.5(109 / 20)$ \\
\hline \hline
\end{tabular}


[lower images in Fig.13]. The feature values were calculated from the extracted candidate regions. The distribution is also shown in Fig.14(a). The Scm and Scar of FPs tend to be lower than those of TPs. Therefore, FPs could be classified by determining appropriate distinction lines. The distinction lines were determined experimentally. Local mean $(\mathrm{Lm})$, local standard deviation $(L s d)$ and local directional variance of pixel gradient ( $L d v p g$ ) were used to eliminate FPs generated by the LWTM. $L m$ and $L s d$ were calculated in the $3 \times 3$ pixel grid that includes the center pixel of the candidate ROI. The distribution is shown in Fig.14(b). It is found that some FPs has lower $L m$ and higher $L s d$. Therefore, FPs could be also classified by determining appropriate distinction lines. Calculation method of $L d v p g$ is similar to that of Dvpg described in Section 2. In this step, after deleting lung wall area, Dvpg $g_{\text {out }}$ was calculated and used as Ldvpg. Compared with Ldvpg of TPs, several FPs had lower values of Ldvpg.

Then, we reconsidered threshold-values of features used in Section 2. Reconsidered features were area, Cont, Mmct and Dvpg. In Section 2, we described that the GATM was performed twice, once using the spherical models and once using the circular models as reference images, and the corresponding threshold-value in each feature for classification of FPs was obtained by the technique. To improve the FP elimination rate, in the current study, different threshold-values were used between the candidates detected by using the spherical models and those detected by using circular models. As an example, distributions of Mmct verses area are shown in Fig.15. The tendency is similar between Fig15(a) and Fig.15(b), however, notice that it is possible to eliminate further FPs by determining the detailed threshold-values.

\section{RESULT AND DISCUSSION}

We applied our scheme to 20 clinical cases (15 abnormal and five normal cases), consisting of 557 slice images, with the $10 \mathrm{~mm}$ slice thickness. A physician detected 98 nodules from the slices in these abnormal cases. The number of detected nodules ranged from one to 20 per case. Table 1 shows our previous results obtained by the scheme described in Section 2. The scheme had detected 71 nodules out of the total 98 with 5153 FPs. Then, through feature analysis using thirteen features, the number of FPs dropped to 616 (30.8/case).

The improved results of FPs elimination are shown in Table 2. It was possible to decrease the number of FPs from 616 to 109 without eliminating TPs. Through this improvement, the number of FPs became 5.5/case while keeping the sensitivity of approximately $72 \%$. The eliminated FPs in this study are shown in Fig.16 as well as Fig.13. Here, in this study, although our CAD performance was improved, many of parameters were determined experimentally using only 20 cases. So, we need more samples of nodular cases in CT images for analytically determining these parameters.

\section{CONCLUSION}

The outlines of our detection scheme based on two template-matching techniques (GATM and LWTM) and feature analysis were described. In the scheme, feature analysis for FPs elimination was improved by adding five features and by reconsidering threshold-values of features in this study. Through the improvement, the number of FPs became 5.5/case from 30.8/case while keeping the sensitivity of approximately $72 \%$. We are now working to collect more cases and to obtain higher sensitivity.

\section{ACKNOWLEDGMENT}

This work was supported in part by a Grant-In-Aid for Scientific Research (Intelligent Assistance for Diagnosis of Multi-dimensional Medical Images) from the Ministry of Education, Culture, Sports, Science and Technology.

\section{REFERENCES}

1. T. Okumura, T. Miwa, J. Kako, S. Yamamoto, M. Matsumoto, Y. Tateno, T. Iinuma and T. Matsumoto, "VariableN-Quoit fiter applied for automatic detection of lung cancer by X-ray CT", Proc. of Computer-Assisted Radiology (CAR'98), 242-247, Tokyo, Japan, 1998.

2. K. Kanazawa, Y. Kawata, N. Niki, H. Satoh, H. Ohmatsu, R. Kakinuma, M. Kaneko, N. Moriyama and K. Eguchi, "Computer-aided diagnosis for pulmonary nodules based on helical CT images", Compt. Med. Imag. Graph., 22(2), 1998, 157-167. 
3. A. G. Armato, M. L. Giger, C. Moran, J. T. Blackburn, K. Doi and H. MacMahon, "Computerized detection of pulmonary nodules on CT scans", Radiographics, 19(5), 1999, 1303-1311.

4. Y. Lee, T. Hara, H. Fujita, S. Itoh and T. Ishigaki, "Nodule detection on chest helical CT scans by using a genetic algorithm”, Proc. of IASTED Intelligent Information Systems, 595-604, Grand Bahama Island, Bahamas, 1997.

5. Y. Lee, A. Kojima, T. Hara, H. Fujita, S. Itoh and T. Ishigaki, "Automated detection of nodular shadows on lung walls for chest helical CT images by using a template matching based on semicircular models", Trans. IEICE., J83D-II(1), 2000, 419-422.

6. Y. Lee, T. Hara, H. Fujita, S. Itoh and T. Ishigaki, "Automated detection of pulmonary nodules in helical CT images based on an improved template-matching technique", IEEE Trans. Med. Imaging, 20(7), 2001, 595-604.

7. Y. Lee, T. Nakagawa, T. Hara, H. Fujita, S. Itoh and T. Ishigaki, “Automated detection of nodules on helical X-ray CT images using higher-order autocorrelation features”, Medical Imaging and Information Sciences, 18(3), 2001, 135-143.

8. M. S. Brown, M. F. McNitt-Gary, J. G. Goldin, R. D. Suh, J. W. Sayre and D. R. Aberle, "Patient-specific models for lung nodule detection and surveillance in CT images", IEEE Trans. Med. Imaging, 20(12), 2001, 1242-1250.

9. L. Fan, C. L. Novak, J. Qian, G. Kohl and D. P. Naidich, "Automated detection of lung nodules from multi-slice low-dose CT images", Proc. of SPIE, 4322, 1828-1935, San Diego, USA, 2001.

10. M. N. Gurcan, B. Sahiner, N. Petric, H. P. Chan, E. A. Kazerooni, P. N. Cascade and L. Hadjiiski, "Lung nodule detection on thoracic computed tomography images: Preliminary evaluation of a computer-aided diagnosis system", Med. Phys. 29(11), 2002, 2552-2558.

11. Q. Li, S. Sone and K. Doi, "Selective enhancement filters for nodules, vessels, and airway walls in two- and threedimensional CT scans", Med. Phys., 30(8), 2003, 2040-2051.

12. K. Suzuki, S. G. Armato, F. Le, S. Sone and K. Doi, "Massive training artificial neural network (MTANN) for reduction of false-positives in computerized detection of lung nodules in low-dose computed tomography", Med. Phys. 30(7), 2003, 1602-1617.

*lee@ clg.niigata-u.ac.jp; phone +81-25-227-0957; fax +81-25-227-0749 